\title{
BMJ Open Efficacy and safety of renal denervation for Chinese patients with resistant hypertension using a microirrigated catheter: study design and protocol for a prospective multicentre randomised controlled trial
}

\begin{abstract}
Zongjun Liu, ${ }^{1}$ Li Shen, ${ }^{2}$ Weijian Huang, ${ }^{3}$ Xianxian Zhao, ${ }^{4}$ Weiyi Fang, ${ }^{5}$ Changqian Wang, ${ }^{6}$ Zhaofang Yin, ${ }^{6}$ Jianan Wang, ${ }^{7}$ Guosheng Fu, ${ }^{8}$ Xuebo Liu, ${ }^{9}$ Jianjun Jiang, ${ }^{10}$ Zhihui Zhang, ${ }^{11}$ Jingbo Li, ${ }^{12}$ Yingmin $\mathrm{Lu},{ }^{13}$ Junbo $\mathrm{Ge}^{2}$
\end{abstract}

To cite: Liu Z, Shen L, Huang W, et al. Efficacy and safety of renal denervation for Chinese patients with resistant hypertension using a microirrigated catheter: study design and protocol for a prospective multicentre randomised controlled trial. BMJ Open 2017;7:e015672. doi:10.1136/ bmjopen-2016-015672

- Prepublication history for this paper is available online. To view these files please visit the journal online (http://dx.doi. org/10.1136/bmjopen-2016015672).

ZL and LS contributed equally.

Received 5 January 2017 Revised 19 May 2017 Accepted 26 May 2017

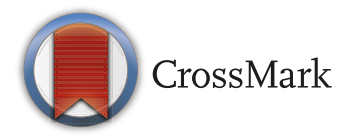

For numbered affiliations see end of article.

Correspondence to

Dr Junbo Ge;

jbge@zs-hospital.sh.cn

\section{ABSTRACT \\ Introduction Available data show that approximately $8 \%-18 \%$ of patients with primary hypertension will develop resistant hypertension. In recent years, catheter- based renal denervation (RDN) has emerged as a potential treatment option for resistant hypertension. A number of observational studies and randomised controlled trials among non-Chinese patients have demonstrated its potential safety and efficacy.}

Methods and analysis This is a multicentre, randomised, open-label, parallel-group, active controlled trial that will investigate the efficacy and safety of a $5 \mathrm{~F}$ saline-irrigated radiofrequency ablation (RFA) used for RDN in the treatment of Chinese patients with resistant hypertension. A total of 254 patients who have failed pharmacological therapy will be enrolled. Eligible subjects will be randomised in a 1:1 ratio to undergo RDN using the RFA plus antihypertensive medication or to receive treatment with antihypertensive medication alone. The primary outcome measure is the change in 24 hours average ambulatory systolic blood pressure from baseline to 3 months, comparing the RDN-plusmedication group with the medication-alone group. Important secondary endpoints include the change in office blood pressure from baseline to 6 months after randomisation. Safety endpoints such as changes in renal function will also be evaluated. The full analysis set, according to the intent-to-treat principle, will be established as the primary analysis population. Ethics and dissemination All participants will provide informed consent; the study protocol has been approved by the Independent Ethics Committee for each site. This study is designed to investigate the efficacy and safety of RDN using a 5F saline microirrigated RFA. Findings will be shared with participating hospitals, policymakers and the academic community to promote the clinical management of resistant hypertension in China.

Trial registration ClinicalTrials.gov ID: NCT02900729; pre-results.
Strengths and limitations of this study

- A randomised controlled trial accords in principle with recommendations by European Expert Group.

- Strict standardisation of antihypertensive medications during the study.

- Enrol Chinese hypertensive patients only, which might affect generability of study findings.

- Failure to implement sham procedure as control might introduce Hawthorne effects.

\section{INTRODUCTION}

Hypertension represents a significant global public health problem, contributing to vascular and renal morbidity, cardiovascular mortality and economic burden. Although there are many methods for treating primary hypertension, more than half of the patients are still unable to achieve their treatment goal. ${ }^{12}$ Available data show that approximately $8 \%-18 \%$ of patients with primary hypertension present with resistant hypertension, ${ }^{3} 4$ defined as a systolic blood pressure (SBP) of $140 \mathrm{~mm} \mathrm{Hg}$ or higher despite adherence to at least three maximally tolerated doses of antihypertensive medications from complementary classes, including a diuretic at an appropriate dose. ${ }^{5}$ Compared with those with controlled blood pressure (BP), patients with resistant hypertension are at greater risk for developing adverse cardiovascular events, leading to an unfavourable prognosis without adequate treatment. ${ }^{6}$ Because of the complex pathophysiology of resistant hypertension, however, there are limited strategies available to treat it efficiently. 
Following the clinical use of radiofrequency ablation (RFA) catheters in recent years, catheter-based renal denervation $(\mathrm{RDN})$ has emerged as a potential treatment option for resistant hypertension. This technique delivers low-level radiofrequency energy throughout the renal artery wall to disrupt renal nerves and thereby modulate BP to some extent. A number of observational studies and randomised controlled trials among non-Chinese patients have demonstrated both the safety and the potential efficacy of this new therapy, ${ }^{7-15}$ whereas several other studies failed to show extra benefits when RDN was applied. ${ }^{16-18}$ Whenever doubts arise concerning the effectiveness of a therapeutic approach, rigorously designed studies are warranted to furnish conclusive evidence. According to the clinical consensus from the European Expert Group, ${ }^{19}$ many factors could affect the results of RDN in clinical trials, including procedural aspects, patient populations and design considerations. Many aspects of the RDN procedure may affect the success of the ablation; furthermore, whether denervation has been completely achieved in a specific patient remains the key factor for the efficacy of RDN.

Together with these key recommendations, ${ }^{19}$ we present the rationale and methodology for a randomised controlled trial of RDN using a $5 \mathrm{~F}$ saline microirrigated
RFA for the treatment of hypertension in Chinese patients who have failed standardised pharmacological therapy.

\section{METHODS/DESIGN \\ Study design}

This trial (ClinicalTrials.gov ID: NCT02900729, Pre-results stage) is a multicentre, randomised, open-label, parallel-group, active controlled trial that will investigate the efficacy and safety of a 5F saline-irrigated RFA used in RDN for the treatment of Chinese patients with resistant hypertension. The RFA catheter under study is manufactured by Shanghai WiseGain Medical Devices. Approximately 13 clinical centres will participate in this trial. A brief flow chart of this trial is provided in figure 1.

\section{Study patients}

A total of 254 patients who have failed pharmacological therapy will be enrolled. The following are the inclusion criteria:

1. Subject with primary hypertension has 24 hours ambulatory SBP $\geq 135 \mathrm{~mm} \mathrm{Hg}$ and office $\mathrm{SBP} \geq 140 \mathrm{~mm}$ $\mathrm{Hg}$ /office diastolic blood pressure (DBP) $\geq 90 \mathrm{~mm} \mathrm{Hg}$ after 4 weeks'standardised triple therapy.

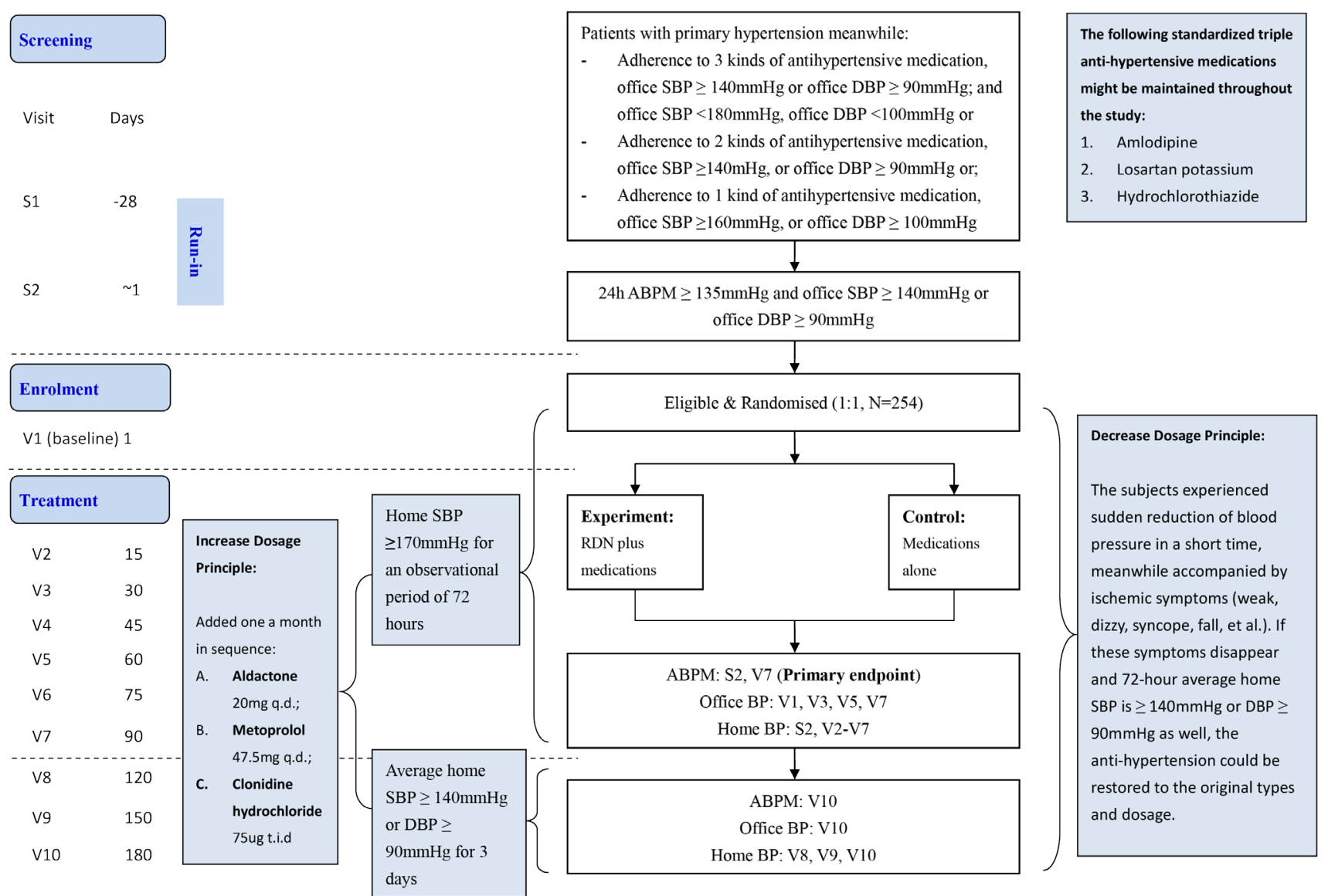

Figure 1 Study flow chart and principles of adjusting antihypertensive medications. ABPM, ambulatory blood pressure monitoring; BP, blood pressure; DBP, diastolic blood pressure; RDN, renal denervation; SBP, systolic blood pressure. 
2. Subject is $\geq 18$ and $<80$ years old at the time of randomisation.

3. Subject agrees to have all study procedures performed and is willing to provide written informed consent to participate in this clinical study.

The exclusion criteria are as follows:

1. Subject has acute or serious systemic infection.

2. Subject has a history of renal artery interventional therapy.

3. Subject lacks suitable renal artery anatomy for percutaneous renal sympathetic nerve RFA surgery, including but not limited to the presence of serious aorta or renal-artery tortuosity or renal-artery stenosis.

4. Subject has experienced a myocardial infarction, unstable angina pectoris, syncope, or a cerebrovascular accident within 3 months of the screening period, or has widespread atherosclerosis, with documented intravascular thrombosis.

5. Subject has aortic dissection aneurysm.

6. Subject has primary pulmonary hypertension.

7. Subject has an estimated glomerular filtration rate of less than $40 \mathrm{~mL} / \mathrm{min} / 1.73 \mathrm{~m}^{2}$ according to the Modification of Diet in Renal Disease formula.

8. Subject had a definite diagnose of coronary heart disease requiring beta-blockers.

9. Subject has class III-IV heart failure or left ventricular ejection fraction $<45 \%$.

10. Subject has atrial fibrillation.

11. Subject has a significant bleeding tendency or blood system disease (s).

12. Subject has a malignancy or end-stage disease(s).

13. Subject has secondary hypertension.

14. Subject has type 1 diabetes mellitus.

15. Subject has other conditions inappropriate for participation, at the investigator's discretion.

16. Subject has a medical ethics issue of concern, at the investigator's discretion, such as presence of an average $\mathrm{SBP} \geq 170 \mathrm{~mm} \mathrm{Hg}$ on 24 hours ambulatory BP monitoring after 4 weeks'standardised triple therapy.

\section{Recruitment process}

Before enrolment, there will be two screening visits. Each participant will be assigned a unique identification number during the first screening visit. In addition to the above-mentioned entry criteria, patients with primary hypertension who meet one of the three following criteria will be considered for further evaluation at the second screening visit:

- Adherence to three kinds of antihypertensive medication: office SBP $\geq 140 \mathrm{~mm} \mathrm{Hg}$ or office DBP $\geq 90 \mathrm{~mm}$ $\mathrm{Hg}$, and office $\mathrm{SBP}<180 \mathrm{~mm} \mathrm{Hg}$, office DBP $<100 \mathrm{~mm}$ $\mathrm{Hg}$.

- Adherence to two kinds of antihypertensive medication: office SBP $\geq 140 \mathrm{~mm} \mathrm{Hg}$ or office DBP $\geq 90 \mathrm{~mm}$ $\mathrm{Hg}$.
Adherence to one kind of antihypertensive medication: office SBP $\geq 160 \mathrm{~mm} \mathrm{Hg}$ or office DBP $\geq 100 \mathrm{~mm}$ $\mathrm{Hg}$.

For any initially eligible patients as mentioned above, three basic kinds of antihypertensive medication, for example, standardised triple antihypertensive medications consisting of amlodipine $5 \mathrm{mg}$ per day, losartan potassium $50 \mathrm{mg}$ and hydrochlorothiazide $12.5 \mathrm{mg}$ per day, will be administered for at least 4 weeks (run-in period). Patients who meet the following BP threshold criteria will then be eligible for randomised assignment after the second screening period: 24 hours ambulatory $\mathrm{BP} \geq 135 \mathrm{~mm} \mathrm{Hg}$ and office SBP $\geq 140 \mathrm{~mm} \mathrm{Hg}$, or office $\mathrm{DBP} \geq 90 \mathrm{~mm} \mathrm{Hg}$.

\section{Randomisation process}

Eligible patients with resistant hypertension will be randomly assigned to one of two study treatment groups in a 1:1 ratio. A stratified block randomisation with randomly varying block size will be performed, stratified according to study site. Random assignment is generated by an independent statistician and implemented via random envelopes assigned to each site. These envelopes are opaque and without any information identifying treatment assignment from appearance. Anyone is prohibited to open an envelope unless there is a real eligible subject requiring randomisation. In order to avoid potential selection bias, the sequence is concealed from both clinical staff and patients until assignment. Hence, neither investigators nor participants can influence which group the study patients are assigned to.

\section{Description of the interventions}

The enrolled subjects will be randomised to undergo RDN using a $5 \mathrm{~F}$ saline microirrigated RFA plus antihypertensive medication, or to be treated with antihypertensive medication alone. RDN will be performed according to the device's instructions for use.

The study patients will be advised to maintain baseline antihypertensive medication in the first 90 days after randomisation. However, the three baseline antihypertensive medications (eg, calcium antagonist, angiotensin II receptor antagonist and diuretics) will be adjusted after randomisation when clinically necessary. Criterion for dosage reduction: subjects experience a sudden reduction in BP within a short time, meanwhile accompanied by ischaemic symptoms (weakness, dizziness, syncope, fall and so on). If these symptoms disappear and 72 hours average home $\mathrm{SBP}$ is $\geq 140 \mathrm{~mm} \mathrm{Hg}$ or $\mathrm{DBP} \geq 90 \mathrm{~mm} \mathrm{Hg}$, the antihypertensive medication may be restored to the original type and dosage. Criterion for dosage increase: if home SBP is $\geq 170 \mathrm{~mm} \mathrm{Hg}$ for an observational period of 72 hours from randomisation through 90 days, or from 91 days through 180 days if average home SBP is $\geq 140 \mathrm{~mm}$ $\mathrm{Hg}$ or DBP is $\geq 90 \mathrm{~mm} \mathrm{Hg}$ based on three consecutive daily measurements, the following three kinds of drugs could be added, one per month in sequence: aldactone $20 \mathrm{mg}$ per day, metoprolol succinate sustained-release tablet 
$47.5 \mathrm{mg}$ per day and clonidine hydrochloride tablets $75 \mu \mathrm{g}$ thrice daily (figure 1 ).

For patients receiving antihypertensive medication alone, after maintenance of baseline standardised triple antihypertensive medications for 90 days postrandomisation and then medically necessary adjustment of antihypertensive medications for another 90 days, subjects will be allowed to crossover to undergo RDN if they still meet the original inclusion criteria for the study.

\section{RDN procedure}

Under local anaesthesia, RDN procedures are to be performed by interventionists at each study site after a unified training session. Following preoperative preparation, the ablation catheter will be advanced to the distal segment of the renal artery through the $7 \mathrm{~F}$ guidance catheter.

The ablation involves at least six applications to each renal artery, according to the length of the artery's main stem. If the main renal artery is less than $15 \mathrm{~mm}$, two ablations should be delivered to the main bifurcation with diameter $>3 \mathrm{~mm}$ in order to ensure six ablation lesions on each side. Treatment begins from the distal end of the artery or the main bifurcation in a helical pattern as the catheter is pulled back.

For every renal artery ostium, the catheter must be manoeuvred to at least one position in each of the distal, middle and proximal segments. The ablation energy will be $8-10 \mathrm{~W}$ in the distal segment, $10-11 \mathrm{~W}$ in the middle segment and $12 \mathrm{~W}$ in the proximal segment. Each ablation will last $60 \mathrm{~s}$. The ideal target outcome is for the energy titration to achieve a $10 \%-20 \%$ drop in impedance at each location. If the drop in impedance is less than $5 \%$, or the ablation energy is unable to achieve the preset wattage, the ablation will be stopped, and the catheter will be repositioned.

\section{Study visits}

Nine study visits will be scheduled following the baseline visit: once every 15 days in the first 90 days and then every 30 days until 180 days. For the 3rd, 5th, 7th and 10th visits, patients will return to the clinic office; for the remaining visits, the patients will be consulted by phone. At every visit, data relating to $\mathrm{BP}$, medication, adverse events, and so on will be collected.

The subjects may withdraw from the study if any of the following conditions occur:

- After 4 weeks postrandomisation, the office or home SBP is $\geq 180 \mathrm{~mm} \mathrm{Hg}$ for more than 1 week while standardised antihypertensive medications are maintained.

- Based on the investigator's discretion, the subject is no longer eligible for the study for any reason.

\section{Outcome measures}

\section{Primary outcome}

The primary outcome of this study is the change in 24 hours average ambulatory SBP from baseline to 3 months compared between the RDN-plus-medication group and the medication-only group. The department of laboratory other than the clinical department at each participating site will undertake the ambulatory blood pressure monitoring (ABPM) during the study period. The ABPM machine will record and report ABPM results automatically. This outcome will be strictly standardised in terms of uniform validated devices, appropriate cuff, identical clinical setting and resting condition prior to $\mathrm{BP}$ measurement after mandatory 1-day stay in participating site and so on.

\section{Secondary outcomes}

1. Change in office systolic/diastolic BP from baseline to 6 months postrandomisation.

2. Incidence of achieving target $\mathrm{BP}$ at 6 months postrandomisation. Target BP is defined as daytime ambulatory BP $<135 / 85 \mathrm{~mm} \quad \mathrm{Hg}$, night-time ambulatory $\mathrm{BP}<120 / 70 \mathrm{~mm} \mathrm{Hg}$, or average 24 hours ambulatory $\mathrm{BP}<130 / 80 \mathrm{~mm} \mathrm{Hg}$.

3. Incidence of substantially adjusting antihypertensive medications at 6 months postrandomisation. A substantial adjustment of antihypertensive medications is defined as any change in the number or type of antihypertensive medications, or a $\geq 50 \%$ dose change in the last 2 weeks with respect to any ongoing antihypertensive medications.

4. Incidence of achieving reductions of $\geq 5 \mathrm{~mm} \mathrm{Hg}$, $\geq 10 \mathrm{~mm} \mathrm{Hg}, \geq 15 \mathrm{~mm} \mathrm{Hg}$ and $\geq 20 \mathrm{~mm} \mathrm{Hg}$ in BP, including ambulatory, office and home BP at 6 months postrandomisation.

\section{Safety endpoints}

The safety endpoints mainly include any adverse events (eg, puncture haematoma, thrombosis, renal artery stenosis and renal artery dissection as adverse event of special interest, and so on), a change in renal function (serum creatinine, urea nitrogen, serum uric acid, creatinine clearance, and so on), other laboratory tests (liver function and serum biochemistry) and cardiovascular complications.

\section{Sample size calculation}

We used R V.3.2.3 (R Core Team. R: A language and environment for statistical computing. Vienna, Austria: R Foundation for Statistical Computing, 2014. http:// www.R-project.org/: last accessed June 2016) to estimate sample size. The trial is designed to compare the difference in average ambulatory SBP as a change from baseline to 3 months between the RDN-plus-medication group and the medication-alone group. With a sample size of 108 randomised patients per group, the betweengroup comparison will be powered at $90 \%$ to establish the superiority of added RDN for the primary endpoint at a two-sided significance level of 0.05 , assuming that the true SBP difference is $8 \mathrm{~mm} \mathrm{Hg}$ with a common SD of $18 \mathrm{~mm} \mathrm{Hg}$. Given an expected dropout rate of $15 \%$ in the first 3 months postrandomisation, a total of 254 patients (127 patients per group) must be enrolled in the study. 
Results of 10000 simulations using this estimated sample size for each study showed that an empirical power of $98 \%$ would be reached for the analysis of the BP target rate $(56 \%$ vs $44 \%)$ as the important efficacy endpoint, using the Cochran-Mantel-Haenszel (CMH) test with antihypertensive medication adjusted or not within the last 2 weeks as stratification factor.

\section{Statistical analysis}

The full analysis set, according to the intent-to-treat principle, will be established as the primary analysis population. A two-sided $\mathrm{p}$ value of $<0.05$ will be considered to indicate significance for any statistical tests. R V.3.2.3 and SAS software V.9.2 will be used for statistical analysis. Such data as demographics, baseline characteristics and safety will be summarised according to treatment group.

The primary efficacy outcomes will be analysed using analysis of covariance (ANCOVA) with treatment group as fixed factor and BP values at baseline as covariate. The sensitivity analysis with stratifying variable centre as a fixed effect of ANCOVA will also be considered as appropriate. The paired and unpaired $t$-tests will further be used to test BP reduction within each group and between groups, respectively. The 95\% CIs for the differences between treatment groups will also be calculated. Subgroup analyses are prespecified according to the following prognostic factors: sex, age, diabetes, body mass index, estimated glomerular filtration rate and aldosterone use at baseline.

Blood pressure target rate at 6 months will be analysed using the CMH test, with antihypertensive medication adjusted or not within the last 2 weeks as stratification factor. Other categorical data will be tested using Pearson's $\chi^{2}$ test or Fisher's exact test, as appropriate. Other continuous efficacy endpoints will be analysed similarly to the primary endpoint. Mixed-model repeated measures analysis including terms for treatment group, time, baseline measurement and time by treatment group interaction will be considered to compare $\mathrm{BP}$ reduction in the study.

\section{DISCUSSION}

The design and methods of this trial satisfy the requirements to test whether a $5 \mathrm{~F}$ saline microirrigated RFA used in RDN is safe and effective for patients who remain hypertensive despite adherence to polypharmacy.

With the recognition of the role of the sympathetic nervous system in the development and progression of hypertension, ${ }^{2021}$ catheter-based RDN has been developed to reduce sympathetic nervous activity and subsequently reduce $\mathrm{BP}$, as well as mortality and morbidity, in patients with uncontrolled hypertension ${ }^{22-25}$ and the prevention of recurrences of atrial fibrillation, ${ }^{26}$ the improvement of glycaemic control ${ }^{27}$ and the mitigation of pulmonary arterial hypertension as well. ${ }^{28}$ However, the clinical evidence in support of RDN as an effective interventional technique in patients with resistant hypertension appears conflicting. Several large studies support both the safety and the efficacy of this new therapy, ${ }^{7-15}$ but some studies failed to show the superiority resulting from added RDN. ${ }^{16-18}$ In view of this controversy, the European Expert Group convened a clinical consensus conference and agreed on recommendations for future randomised controlled trials of RDN in hypertension. The design and methods of our trial accord in principle with the recommendations.

The RDN procedure is so complex that the efficacy of ablation may be influenced by many factors, such as renal artery anatomy, the depth of the ablation lesion, atherosclerosis, and so on. Achieving complete ablation will pose a challenge to the operator, the equipment and the procedure. A study of the anatomic assessment of sympathetic peri-arterial renal nerves showed that the greatest number of nerves were observed in the proximal and middle segments of the renal artery, while the smallest number were seen in the distal segment. However, in the main renal artery, the distance from the nerve to the renal artery lumen is shorter than in the proximal and middle segments, being approximately $4.28 \mathrm{~mm} .{ }^{29}$ Another study showed that, for a patient with atherosclerosis, the RFA-induced damage did not penetrate deeper than $2 \mathrm{~mm}$ from the luminal surface, leaving unaffected a large part of the nerves in (peri-) adventitial areas remote from the vascular lumen. ${ }^{30}$ An animal study showed that the ablation zone geometries varied in arc, area and depth, depending on the composition of the adjacent tissue substructure. ${ }^{31}$ In addition, the delivered power density was influenced by tissue substructure and peaked at the conductivity discontinuities between soft fatty adventitia and water-rich tissues, not at the electrode-tissue interface. ${ }^{31}$ With a greater recognition of nerve distribution, the ablation depth and location should be taken carefully into account.

In previous studies, a non-irrigated catheter was usually used, and the ablation energy was usually $8 \mathrm{~W}$. Increasing ablation energy or prolonging ablation time could make the ablation deeper. However, using a non-irrigated catheter could raise the temperature of the luminal surface too much to increase the ablation power. In this study, radiofrequency energy delivery with the use of cold saline irrigation seems safe and effective. By actively cooling the ablation electrode during RFA, it is possible to minimise the possibility of char formation and also decrease the probability of vasospasm. These advantages to saline irrigation are so significant that most cardiac ablations are now performed using irrigated ablation catheters. ${ }^{32}$ Ahmed et al, in a small single-arm study, demonstrated that RND can be performed safely and effectively using a saline-irrigated RFA in patients with hypertension. ${ }^{33}$ Using a saline-irrigated catheter, with the protection of cold saline, higher ablation energy can be delivered, ensuring the ablation depth. Indeed, the saline-irrigated catheter has been widely used in cardiac ablation.

In most clinical trials involving RDN, adrenal artery less than $4 \mathrm{~mm}$ in diameter could not be ablated because of 
the limited operation equipment. In this study, the $5 \mathrm{~F}$ saline microirrigated RFA catheter is smaller and more flexible, so it can be used in renal arteries with diameter $<4 \mathrm{~mm}$, while minimising the possibility of peripheral artery-related complications.

In this study, the operation procedure will also be unified. A similar spiral ablation will be used and at least one site must be ablated at each of the distal segments of the renal artery, the middle segment, proximal and opening. Four quadrants will be ablated. There are a total of six ablation points on each side of the renal artery. The ablation energy will also be standardised to ensure sufficient ablation.

In this trial, patients with 24 hours ambulatory SBP $\geq 135 \mathrm{~mm} \mathrm{Hg}$ and office SBP $<170 \mathrm{~mm} \mathrm{Hg}$ will be eligible for enrolment, while patients with high-risk characteristics will be excluded. Given this restriction, the patients enrolled in this study will mostly have mild to moderate hypertension and might be more responsive to RDN-induced changes in sympathetic tone. In addition, it will be safer for these patients to strictly follow a standardised medication regimen. Moreover, higher drug adherence will be expected in this study, because of the lower level of discomfort occurring in the management of mild to moderate rather than severe hypertension.

In the study period, the antihypertensive medications administered are explicitly specified: standardised triple antihypertensive medications include a calcium channel blocker, a renin-angiotensin system blocker and a diuretic. In the Symplicity HTN-3 study, ${ }^{16}$ the maximum doses were administered, and 39\% patients required medication adjustment because of adverse events; this may be related to the negative conclusions of that study. Conversely, in the DENERHTN study, ${ }^{15}$ the antihypertensive medications in the RDN group and control group were strictly regulated, and the study results supported the superiority of RDN. The rigorous specification of medication may be an important factor influencing the study results.

For this study, ambulatory BP is used as the primary endpoint and office BP as the secondary parameter. In fact, several previous studies have documented a better prognostic value of ambulatory over office BP in different populations. ${ }^{34-38}$ Among the previous trials conducted on RDN, only the DENERHTN study ${ }^{15}$ successfully used the change in mean daytime ambulatory SBP as primary endpoint and that study found RDN to have superior efficacy. The Expert Group also strongly recommended ambulatory $\mathrm{BP}$ as the primary measure of response to RDN. Using ambulatory BP monitoring to measure efficacy could exclude pseudo-resistance due to a "whitecoat' effect.

There is also one limitation regarding the selection of the control group. Because of the poor acceptability by patients in our routine clinical practice and potential ethical problems, a sham operation will not be performed in this study; its omission might thus be a potential confounder for study outcomes. Although a sham procedure could reduce some Hawthorne effects, it could not eliminate other biases that are considered as reasons for the lack of benefit from RDN.

\section{ETHICS AND DISSEMINATION}

This trial will be conducted in accordance with the principles outlined in the Declaration of Helsinki and will follow the Consolidated Standards of Reporting Trials (CONSORT) statement (http://www.consort-statement.org/). It has been approved by the Independent Ethics Committee for each site (approval no 2016-46). All subjects will be required to sign a written informed consent document before their participation in the trial.

This study is designed to investigate the efficacy and safety of RDN using a 5F saline-irrigated RFA in Chinese patients with hypertension who are resistant to medication therapy. Its goal is to provide clinical evidence that RDN with a 5F saline-irrigated RFA is both safe and effective in Chinese patients with drug-resistant, systemic hypertension. Findings will be shared with participating hospitals, policymakers and the academic community to promote the clinical management of resistant hypertension in China.

\section{Trial status}

The study enrolled the first patient in March 2017 and is expected to finish patient enrolment within 1.5 years.

\section{Author affiliations}

${ }^{1}$ Department of Cardiology, Putuo Hospital affiliated to Shanghai Traditional Chinese Medicine University, Shanghai, China

${ }^{2}$ Department of Cardiology, Zhongshan Hospital affiliated to Fu Dan University, Shanghai, China

${ }^{3}$ Department of Cardiology, The First Hospital affiliated to Wenzhou Medical College, Wenzhou, China

${ }^{4}$ Department of Cardiology, Changhai Hospital affiliated to Second Military Medical University, Shanghai, China

${ }^{5}$ Department of Cardiology, Shanghai Chest Hospital affiliated to Shanghai Jiao Tong University, Shanghai, China

${ }^{6}$ Department of Cardiology, Shanghai Ninth People's Hospital affiliated to Shanghai Jiao Tong University, Shanghai, China

${ }^{7}$ Department of Cardiology, The Second Hospital affiliated to Zhejiang University, Hangzhou, China

${ }^{8}$ Department of Cardiology, Sir Run Run Shaw Hospital affiliated to Zhejiang University, Hangzhou, China

${ }^{9}$ Department of Cardiology, Tongji Hospital affiliated to Tongji University, Shanghai, China

${ }^{10}$ Department of Cardiology, Taizhou Hospital, Taizhou, China

${ }^{11}$ Department of Cardiology, The Third Xiangya Hospital of Central South University, China

${ }^{12}$ Department of Cardiology, Shanghai Sixth People's Hospital affiliated to Shanghai Jiao Tong University, Shanghai, China

${ }^{13}$ Department of Cardiology, Xinhua Hospital affiliated to Shanghai Jiao Tong University School of Medicine Chongming Branch, Shanghai, China

Acknowledgements The authors wish to thank May Dong (MD) and Sam Zhong (SZ) for their generous assistance with this study.

Contributors ZL, LS and JG conceived and designed the study. ZL, LS and SZ supervised the power analyses and wrote the data analysis section. ZL and JG bear overall responsibility for the design, ethical conduct and publication of the study. Administrative, technical and material support was provided by ZL and LS. All authors are involved in the protocol discussion, and they will take responsibility for study data gathering and verification. All authors edited the draft and contributed substantially to the manuscript; they all approved this submission. 
Funding This study was funded and conducted by Shanghai WiseGain Medical Devices $C_{0}$., Ltd, which also manufactures the catheters under study.

Competing interests None declared.

Ethics approval Independent ethics committee for each site.

Provenance and peer review Not commissioned; externally peer reviewed.

Data sharing statement There is no additional unpublished data from the study available now.

Open Access This is an Open Access article distributed in accordance with the Creative Commons Attribution Non Commercial (CC BY-NC 4.0) license, which permits others to distribute, remix, adapt, build upon this work non-commercially, and license their derivative works on different terms, provided the original work is properly cited and the use is non-commercial. See: http://creativecommons.org/ licenses/by-nc/4.0/

(c) Article author(s) (or their employer(s) unless otherwise stated in the text of the article) 2017. All rights reserved. No commercial use is permitted unless otherwise expressly granted.

\section{REFERENCES}

1. Lloyd-Jones D, Adams RJ, Brown TM, et al. WRITING GROUP MEMBERS. Heart disease and stroke statistics--2010 update: a report from the American Heart Association. Circulation 2010;121:e46-e215.

2. Wolf-Maier K, Cooper RS, Kramer H, et al. Hypertension treatment and control in five European countries, Canada, and the United States. Hypertension 2004;43:10-17.

3. Mancia G, Fagard R, Narkiewicz K, et al. 2013 ESH/ESC guidelines for the management of arterial hypertension: the Task Force for the Management of Arterial Hypertension of the European Society of Hypertension (ESH) and of the European Society of Cardiology (ESC). Eur Heart J 2013;34:2159-219.

4. Judd E, Calhoun DA. Apparent and true resistant hypertension: definition, prevalence and outcomes. J Hum Hypertens 2014;28:463-8.

5. Sarafidis PA, Georgianos P, Bakris GL. Resistant hypertension--its identification and epidemiology. Nat Rev Nephrol 2013;9:51-8.

6. Vega J, Bisognano JD. The prevalence, incidence, prognosis, and associated conditions of resistant hypertension. Semin Nephrol 2014;34:247-56.

7. Esler MD, Krum H, Schlaich M, et al. Renal sympathetic denervation for treatment of drug-resistant hypertension: one-year results from the Symplicity HTN-2 randomized, controlled trial. Circulation 2012;126:2976-82.

8. Esler MD, Krum H, Sobotka PA, et al. Renal sympathetic denervation in patients with treatment-resistant hypertension (The Symplicity HTN-2 Trial): a randomised controlled trial. Lancet 2010;376:1903-9.

9. Krum H, Schlaich M, Whitbourn R, et al. Catheter-based renal sympathetic denervation for resistant hypertension: a multicentre safety and proof-of-principle cohort study. Lancet 2009;373:1275-81.

10. Mahfoud F, Ukena C, Schmieder RE, et al. Ambulatory blood pressure changes after renal sympathetic denervation in patients with resistant hypertension. Circulation 2013;128:132-40.

11. Persu $A$, Jin $Y$, Azizi $M$, et al. Blood pressure changes after renal denervation at 10 European expert centers. J Hum Hypertens 2014;28:150-6.

12. Worthley SG, Tsioufis CP, Worthley MI, et al. Safety and efficacy of a multi-electrode renal sympathetic denervation system in resistant hypertension: the EnligHTN I trial. Eur Heart J 2013;34:2132-40.

13. Sievert $\mathrm{H}$, Schofer J, Ormiston J, et al. Renal denervation with a percutaneous bipolar radiofrequency balloon catheter in patients with resistant hypertension: 6-month results from the REDUCE-HTN clinical study. Eurolntervention 2015;10:1213-20.

14. Ott C, Mahfoud F, Schmid A, et al. Renal denervation in moderate treatment-resistant hypertension. J Am Coll Cardiol 2013;62:1880-6.

15. Azizi M, Sapoval M, Gosse P, et al. Optimum and stepped care standardised antihypertensive treatment with or without renal denervation for resistant hypertension (DENERHTN): a multicentre, open-label, randomised controlled trial. Lancet 2015;385:1957-65.
16. Bhatt DL, Kandzari DE, O'Neill WW, et al. A controlled trial of renal denervation for resistant hypertension. $N$ Engl J Med 2014;370:1393-401.

17. Fadl Elmula FE, Hoffmann P, Fossum E, et al. Renal sympathetic denervation in patients with treatment-resistant hypertension after witnessed intake of medication before qualifying ambulatory blood pressure. Hypertension 2013;62:526-32.

18. Fadl Elmula FE, Hoffmann P, Larstorp AC, et al. Adjusted drug treatment is superior to renal sympathetic denervation in patients with true treatment-resistant hypertension. Hypertension 2014;63:991-9.

19. Mahfoud F, Böhm M, Azizi M, et al. Proceedings from the European clinical consensus conference for renal denervation: considerations on future clinical trial design. Eur Heart J 2015;36:2219-27.

20. Smith PA, Graham LN, Mackintosh AF, et al. Relationship between central sympathetic activity and stages of human hypertension. Am J Hypertens 2004;17:217-22.

21. Parati G, Esler M. The human sympathetic nervous system: its relevance in hypertension and heart failure. Eur Heart $J$ 2012;33:1058-66.

22. Böhm M, Linz D, Ukena C, et al. Renal denervation for the treatment of cardiovascular high risk-hypertension or beyond? Circ Res 2014;115:400-9.

23. Tsioufis C, Mahfoud F, Mancia G, et al. What the interventionalist should know about renal denervation in hypertensive patients: a position paper by the ESH WG on the interventional treatment of hypertension. Eurolntervention 2014;9:1027-35.

24. Smithwick RH. Surgical treatment of hypertension. Am J Med 1948;4:744-59.

25. Smithwick RH, Thompson JE. Splanchnicectomy for essential hypertension; results in 1,266 cases. J Am Med Assoc 1953;152:1501-4.

26. Pokushalov E, Romanov A, Corbucci G, et al. A randomized comparison of pulmonary vein isolation with versus without concomitant renal artery denervation in patients with refractory symptomatic atrial fibrillation and resistant hypertension. J Am Coll Cardiol 2012:60:1163-70.

27. Mahfoud F, Schlaich M, Kindermann I, et al. Effect of renal sympathetic denervation on glucose metabolism in patients with resistant hypertension: a pilot study. Circulation 2011;123:1940-6.

28. Santos-Gallego CG, Badimón JJ. Catheter-based Renal Denervation as a Treatment for Pulmonary Hypertension: Hope or Hype? Rev Esp Cardiol 2015;68:551-3.

29. Sakakura K, Ladich E, Cheng Q, et al. Anatomic assessment of sympathetic peri-arterial renal nerves in man. J Am Coll Cardiol 2014;64:635-43.

30. Vink EE, Goldschmeding R, Vink A, et al. Limited destruction of renal nerves after catheter-based renal denervation: results of a human case study. Nephrol Dial Transplant 2014;29:1608-10.

31. Tzafriri AR, Keating JH, Markham PM, et al. Arterial microanatomy determines the success of energy-based renal denervation in controlling hypertension. Sci Trans/ Med 2015;7:285ra65.

32. Vest JA, Seiler J, Stevenson WG. Clinical use of cooled radiofrequency ablation. J Cardiovasc Electrophysiol 2008;19:769-73.

33. Ahmed H, Neuzil P, Skoda J, et al. Renal sympathetic denervation using an irrigated radiofrequency ablation catheter for the management of drug-resistant hypertension. JACC Cardiovasc Interv 2012;5:758-65.

34. Redon J, Campos C, Narciso ML, et al. Prognostic value of ambulatory blood pressure monitoring in refractory hypertension: a prospective study. Hypertension 1998;31:712-8.

35. Mancia G, Bombelli M, Facchetti R, et al. Long-term risk of sustained hypertension in white-coat or masked hypertension. Hypertension 2009;54:226-32.

36. Mancia G, Parati G. Ambulatory blood pressure monitoring and organ damage. Hypertension 2000;36:894-900.

37. Mancia G, Parati G. Office compared with ambulatory blood pressure in assessing response to antihypertensive treatment: a metaanalysis. J Hypertens 2004;22:435-45.

38. Mancia G, Zanchetti A, Agabiti-Rosei E, et al. Ambulatory blood pressure is superior to clinic blood pressure in predicting treatmentinduced regression of left ventricular hypertrophy. SAMPLE Study Group. Study on Ambulatory Monitoring of Blood Pressure and Lisinopril Evaluation. Circulation 1997;95:1464-70. 